\title{
Health Monitoring using Edge Cognitive Computing Based Smart Health Care
}

\author{
Gauri D. Kumavat, Santosh Kumar
}

\begin{abstract}
With an enhancement in technology and development of sensors, they have decided to concern the latest technology in particular areas to increases the Quality of their life. One of the important section of research that has seen an agreement of the latest technology is the healthcare. the people who need healthcare services they have to suffer for long process, to reduce the problem As a result, basically this complete technology would try to resole the healthcare issues.

The main goal of the project is to develop a remote healthcare system. It have three main parts. The first and most important section in this process is to detecting the patient current health status using this sensors.

second important thing is sending data to cloud storage and the last section is to get the detected data for remote viewing. Remote viewing of the data provided to the doctor will able to monitor a patients health progress aboard from hospital places.

The Edge-Cognitive- Computing-based (ECC-based) smart healthcare system. This technology is able to check also distinguish and detect the current status of patient using this sensors. It also accommodate the computing resource appropriation of the complete edge computing network expansive to the health different percentage of each user. The experiments show that the ECC-based healthcare system provide a exceed user background and try to improvement the computing resources moderately, as well as consequentially improving in the durability rates of patients in emergency.
\end{abstract}

Index Terms-Cognitive computing, Edge computing, Health care

\section{INTRODUCTION}

A Remote health monitoring system is an expansion of a hospital medical system where a patients vital body can be analyzed. to detect the patient disease is quite complex and high power consuming which required extra time. Continuously increasing the technology to words healthcare industry have led to sensors and micro controllers that are small in their size, quickly get access in different operation, it uses very low power and affordable in cost using different sensors doctor can get the physical status of patient and using those information doctor will able to know disease pattern easily using edge cognitive computing.

It also accommodate the computing resource allocation of the complete edge computing network exhaustive according to the health-risk grade of each individual user. The traditional healthcare systems can be divided into three layers

1. The collection layer

2. The transmission layer

3. The analysis layer
In collection layer first analyzed physical health of patient through sensors and sensor will sensing the data as specified frequency (threshold value).using data mining and machine learning algorithm it is possible to store the data in cloud using internet and analyze the complete information of patient. Finally after completing the process of system it will get the current health status of the users, and takes the appropriate action to that particular patient and provide medical service to the patient.

Remote health monitoring It can provide physical data in the home. This monitoring is better for older patient ill unable to reaches the hospital and take all the facility to keep them self away from hospitals.

Basically Healthcare Sensors are used to collection of the different real time data from patient body and transmit those data or singles to specified cloud.A sensor will analyze the complete program with process in this project using different sensors according what you want to detect and design algorithms to realize your detection. Examples are temperature sensor, heartbeat sensor, humidity sensor.

Edge Cognitive Computing based smart healthcare system can able to analyze and monitor the physical health of patient with cognitive computing using remote health monitoring. The proposed system acquire the accordant health risk grade of the patient according to disease under different health statuses in recondition to a health risk analysis. It adjusts the computing resource distribution and collaborate the complete edge computing network to the health risk condition of each patient, and allow the network to have a patient oriented cognitive intelligent. ECC-based smart healthcare system based on different computing resources and data, and define best use full technology for particular patient. Complete run time execution and their allocation of different resource's are completely based on current health status of patient and also this system able to solve the switching issue of cloud based patient current health cognition.

\section{ECC-BASED SMART HEALTHCARE SYSTEM ARCHITECTURE}

\section{A. Motivation}

In this Medical trade it is very important to to analyze the risk of lacking the data also encountered with the data affinity and pattern recognition. If sometimes the important information has been misplace or misunderstood by someone the patient as well doctor will hard to suffer even death also
Revised Manuscript Received on November 08, 2019.

Gauri D. Kumavat, Department of Computer Engineering, Sandip Institute of Technology and Research Center, Nashik,

Santosh Kumar, Department of Computer Engineering Sandip Institute of Technology and Research Center Nashik 
In this remote health monitoring system will having some scenarios. 1. Patient should having a knowledge about their medical condition with unstable diseases pattern also monitor the different changes in body. If required new medicines will be add-on on than particular treatment.

2.In another case if particular patient suffer from heart attacks which is already have suffer before then system will recognize the complete information and also monitor the current status and generate the alert in before. it will help to prevent the diseases.

\section{Crucial body condition.}

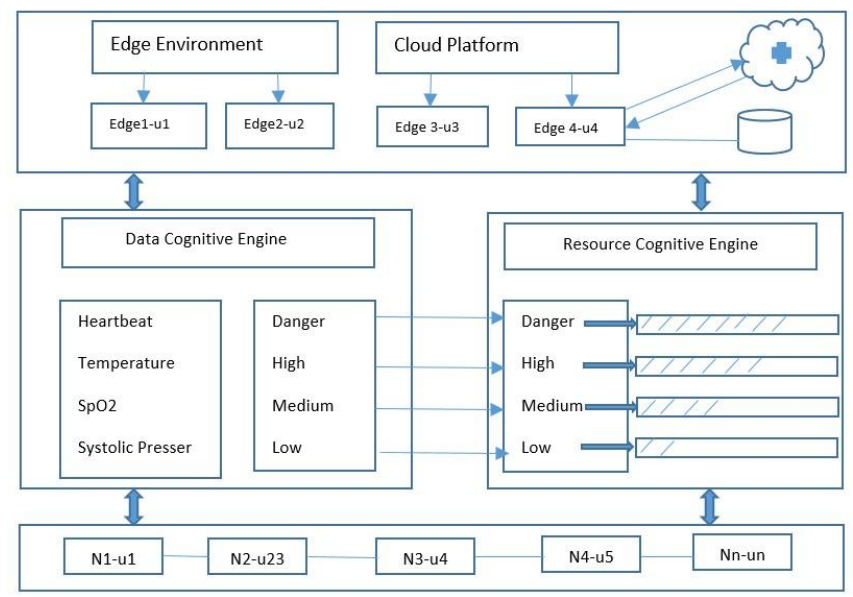

Fig. 1. Architecture of Health Monitoring

ECC-based Smart Healthcare System. It indicates the two different scenarios

\section{Normal case}

\section{Abnormal case}

In normal case patient 1 is connected to the Edge node i.e. ED1 and also its directly connected to the patient 2,3 and another patient i.e. patient no 4 is connected to the Edge 2 i.e. ED2.

In Abnormal or Emergency case suddenly patient no 4 suffer from heart attack then this system will analyzes the current situation and then decide to distribute the patient to another resource that time patient 1,2 and 3 will goes to the Edge 1

i.e.ED1 and Edge 2 i.e. ED2 separately handle patent no4 and patient 4 will get the all services.

The ECC-based smart healthcare system affects the different data resources and different cognition and also get the highly efficient energy, low cost consumption, then and also satisfy the (QoE) Quality of Experience for patient

\section{ECC-BASED SMART HEALTHCARE SYSTEM ENGINE}

\section{A. Data Cognition Engine this cognition Engine} collect the data from external cognition resource and it includes the current body situation i.e. physical signal. The cognitive engine accumulate the internal body data using this technology with having different services and their data flow their network type and also check the communication quality and other different activity like environmental things from different resource.
It significantly the complete big data through machine learning mechanism and deep learning mechanism. it includes cognitive data, explicit data, implicit data analyzes the surrounding apprehension and their patient cognition to encounter the require of different technology or application. For example it illustration analyzes the different activity of particular through the cognition of patient and their daily behaviour, the main section it is edge cognitive their different environment is compatible to realizing the actual or real time service and also user will continuously get the different services according to their diseases start to improve the QoE.

While deliberate multiple users asset collection and their subordinate. which can handle in in multiple environment. In this case patient will get all the facility with highest priority and fastest network resources. In this complete process their are some terminology's to take care the basic data about the patient and their disease pattern the risk levels and also update to the patient actual time and collaborate the run time network resource data in edge cognitive system and their environment. This will provide the high edge computing resource to patient and improve the treatment of patient.

\section{B. Resource Cognition Engine}

This Resource cognition engine will give authority to cognitive compute to access the computing resources communication resources, other than network resources. Then This Resource cognition associate real time data to the data cognitive. after that it can able to receive the analyzed result to the data cognitive engine and able achieve real time dynamic allocation it can take advantage of the network software and its technology it having software define network, virtualization, network slicing, ultralow latency, high flexibility, reliability.

It able to utilize and work on cloud platform using rational algorithm to formation of the diff rent resources and consume the energy and also deal with the increasing the user QoE.

\section{SYSTEM DATA FLOW OF ECC-BASED SMART HEALTHCARE}

\section{A. Explicit Data}

In this data source environment it provides the large amount of explicit data which is undefined and this data includes physical health signs patient core information under the observation and day to day action with their behaviour also check their regular routine like proper diet of particular patient.

In that data cognitive engine the explicit data allude to analyzed and data analysis and processing by using data cognition engine. e.g at a same time machine learning and deep learning, the external data flow are afford the dynamic changes of the implicit data flow.

\section{B. Implicit Data}

In this real-time data flow the dynamic environment contains different resources and their outcomes it include ED Edge cognitive computing resources. 
Communication resources, network resources. (e.g. different services data flow, communication and check the quality other than runtime and network type) and real-time patient data (e.g. real-time disease risk level of the patient and day to day habits or behavior related information). The implicit data run time lead the network related resource divide and is continuously updated in actual health status patient health .

\section{KEY TECHNOLOGIES FOR ECC-BASED SMART HEALTHCARE SYSTEM}

\section{A. Algorithm of Machine Learning}

Basically machine learning is used to cognitive knowledge as its required for the cognitive system. For this issue they having an solution with method of data driven and structure with supervise and unsupervised learning it encounter with certain cognition knowledge.

\section{B. Algorithm of Deep Learning}

In deep learning this involve the complete process of people to the explicit domain the visual and acoustic senses. It should be be based on the big data

The data level constantly in assure the accuracy of the learning algorithm. Virtualization as on that timing deep learning has affected in terms of multimedia data processing and (NLP)i.e. Natural language processing.

\section{SENSOR AND MODULE}

\section{A. Raspberry Pi}

Latest version of Raspberry is raspberry Pi 3 Model B The It doesn't have typical circuit or specific simulation and is a small sized and simple machine.

when you plugged in to all cable it become easy to user to connect on the pi it it will download NOOBS after download is done they will show you some rules after following those rules it will directly connect to the installation process to operating the system on pi. The complete installation is quite easy for all operating systems. it will compatible to all operating system as of it shows the list of all operating system. It is more familiar to desktop laptop as well. the Raspbian is very much familiar to operating system and it is constantly improve now it having some feature to playing games which is related to education background and also add some of programming tools

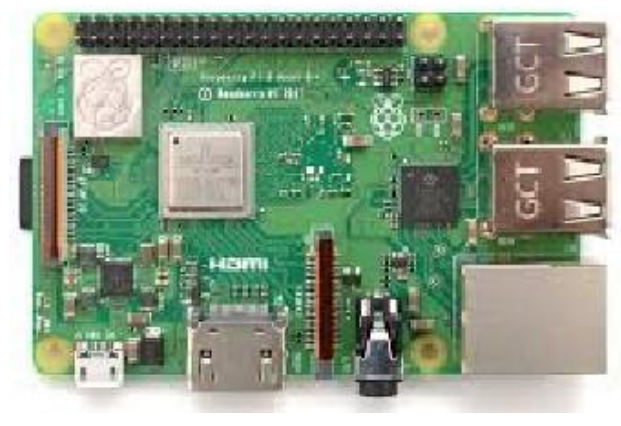

Raspberry Pi

\section{B. GSM Module}

GSM Used to communicate between gsm Module to computer GSM stands for Global system for Communication. In different industry GSM is mostly used for mobile communication. They having extension that is (GPRS) Global Packet Radio Service which is used of GSM extension it allow higher data transfer rate. it having power supply circuit and also communication interface for desktop. GSM model is wireless device that are specially made with GPRS and GSM and Different networks it required (Subscriber Identity Module) just line noraml cell phone to activate communication purpose with networks another important thing they having IMEI (International Mobile Equipment Identity) for cell phone identification number. This GSM model perform different below task.

1. Send and Receive or we can Delete the SMS in particular Sims card.

2.Add or Read And search complete phone memory contacts of the SIM

3. We can also receive or Reject the incoming calls

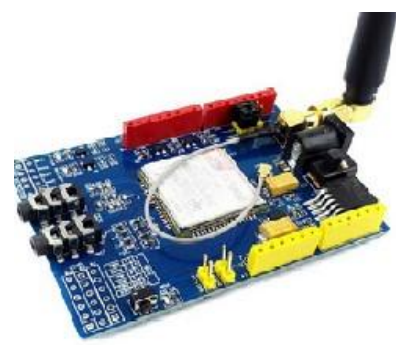

Fig. 3. GSM Module

\section{Temperature Sensor}

Temperature Sensor basically used to measure the temperature. If temperature goes beyond on threshold value it gives alert massage too the particular doctor. Here we are using LM35 sensor which will help to the calculating the temperature value of different patient.LM35 is a IC temperature sensor with its output directly proportional to the temperature in ( $\mathrm{C}$ ) and most important feature it has it calculate the temperature with more accuracy. It also obsessed low heating and does not cause more than $0.1 \mathrm{C}$ temperature. The temperature range if starts from $-55 \mathrm{C}$ to $150 \mathrm{C}$.

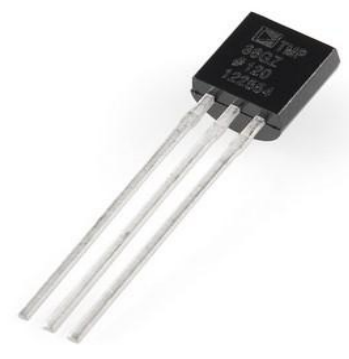

Fig. 4. Temperature Sensor

\section{Humidity Sensor}

In Humidity Sensor which check the Humidity in air and it also measure the moisture and temperature in the air. if temperature is warmer the it 
catches more moisture and it basically used electricity and their capacity electric capacity and their different field also created electric field between them.this sensor composed between with this two metal plates and create conductive polymer which is non conductive polymer this polymer is responsible to check

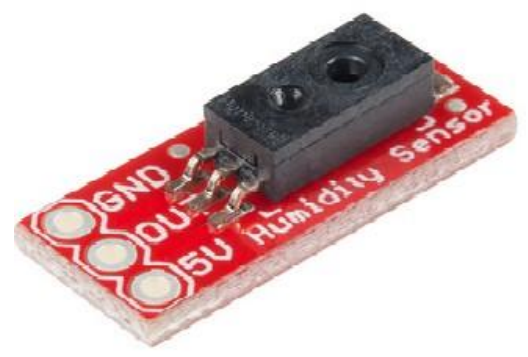

Fig. 5. Humidity sensor

\section{E. Heartbeat Sensor}

Heartbeat sensor gives easy way to study of different Operations of Heartbeat which calculate the beats of human body based of the calculated temperature of patient. How does it calculated? it all basis of blood circulation which is by calculate from the human body it it calculated by finger. while calculating it blink the red light a small LED(Light Emitting Diode) and it transfer the light in Light Dependent Resistor (LDR). this sensor accumulated with LM358-AMP foe analyzing the the heartbeat pulse of human body.

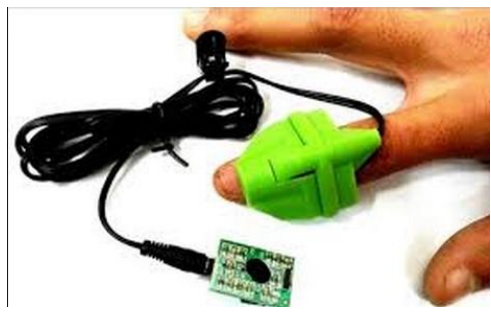

Fig 6 . Heartbeat Sensor

\section{VII. SYSTEM IMPLEMENTING}

In this section the performance of ECC based smart healthcare system it has created a test platform for multi user and their different resources allocation and problem in healthcare scenario It analyze performance of medical institution and experiment.

ECC-based smart healthcare system having cretin conditions which introduce below in different areas. they having user side and edge side the cloud side.

\section{A. Healthcare Scenarios}

ECC-based smart healthcare system having cretin conditions Which introduce below in different areas. They having user side and edge side the cloud side. User side (Data collection layer) In this sect ion when user collect some real time data from sensors. And the sensors

are Temperature sensor, humidity sensor, and heartbeat sensor after taking all the data from user it will directly connected through the server and appropriately to the mobile phones as well because whatever the data received from sensor it goes to the server also shown to the mobile phone as well

- Edge computing side (analysis layer): In this section whatever the data has been collected from the user using sensor the edge communing noted will be analyzed it and process it and also distribute it to the different computing resource with their health status. In case of emergency it send alarm to the hospital as well as doctor for according to their current health situations also send to the regarding person because of this reason the doctor would easily understand the situation of patient .

- Cloud platform side (storage layer):

The cloud side which is basically for providing the different services to the user here we are using for storage purpose and real time handling of currant patient data the compete cloud platform handling process manage by hospital store the basic information about patient it also having medical history of that patient which will help to doctor and as well as patient to find out exact situation of patient it include age of patient, number of diseases and the nature of disease after all collecting the information

B. Dynamic Resource Distribution Mechanism 1 User Command Set

\begin{tabular}{|c|c|c|c|c|c|c|}
\hline \multicolumn{2}{|c|}{ Risk evaluation } & \multicolumn{2}{|c|}{ Low } & \multirow{2}{*}{\begin{tabular}{|l|} 
Medium \\
{$[71-84]$} \\
\end{tabular}} & \multirow{2}{*}{$\begin{array}{l}\text { High } \\
\geq 85\end{array}$} & \multirow{2}{*}{$\begin{array}{l}\text { Danger } \\
-\end{array}$} \\
\hline Basic & Age (year) & - & {$[61-70]$} & & & \\
\hline \multirow{2}{*}{$\begin{array}{c}\text { information } \\
\text { and medical } \\
\text { record }\end{array}$} & $\begin{array}{l}\text { Nature of dis- } \\
\text { ease }\end{array}$ & Recovery & Chronic & Acute & Acute & - \\
\hline & $\begin{array}{l}\text { Numbers of } \\
\text { underlying } \\
\text { disease }\end{array}$ & 0 & 1 & 2 & 3 & - \\
\hline \multirow{4}{*}{$\begin{array}{c}\text { Physiologic } \\
\text { indicator }\end{array}$} & $\begin{array}{l}\text { Respiration } \\
\text { rate (/minute) }\end{array}$ & [12-20] & $\begin{array}{l}{[21-} \\
25] \quad \text { or } \\
{[10-11]}\end{array}$ & {$[26-35]$} & $\begin{array}{l}{[36-50]} \\
\text { or }[6-9]\end{array}$ & $\begin{array}{l}>50 \text { or } \\
\leq 5\end{array}$ \\
\hline & $\begin{array}{l}\text { Heartbeat } \\
\text { (/minute) }\end{array}$ & {$[60-90]$} & $\begin{array}{l}91- \\
100] \text { or } \\
{[51-59]}\end{array}$ & $\begin{array}{l}{[101-} \\
140] \text { or } \\
{[41-50]}\end{array}$ & $\begin{array}{l}{[141-} \\
160] \text { or } \\
{[30-40]}\end{array}$ & $\begin{array}{l}>160 \\
\text { or }<30\end{array}$ \\
\hline & $\begin{array}{l}\text { Temperature } \\
\left({ }^{\circ} \mathrm{C}\right)\end{array}$ & $\begin{array}{l}{[36.0-} \\
37.0]\end{array}$ & $\begin{array}{l}{[37.1-} \\
37.5] \text { or } \\
{[35.5-} \\
35.9]\end{array}$ & $\begin{array}{l}37.6- \\
38.9] \text { or } \\
{[35.0-} \\
35.5]\end{array}$ & $\begin{array}{l}{[39.0-} \\
39.9] \text { or } \\
<35.0\end{array}$ & $\geq 40.0$ \\
\hline & $\begin{array}{l}\text { Systolic pres- } \\
\text { sure (mmHg) }\end{array}$ & [89-139] & $\begin{array}{l}{[140-} \\
159]\end{array}$ & \begin{tabular}{|l}
{$[160-$} \\
$179]$ or \\
{$[85-89]$}
\end{tabular} & $\begin{array}{l}{[180-} \\
189] \text { or } \\
{[80-84]}\end{array}$ & $\begin{array}{l}\geq 190 \\
\text { or }<80\end{array}$ \\
\hline
\end{tabular}

Fig.7.Evaluation Reference.

\section{Typical flow scenario}

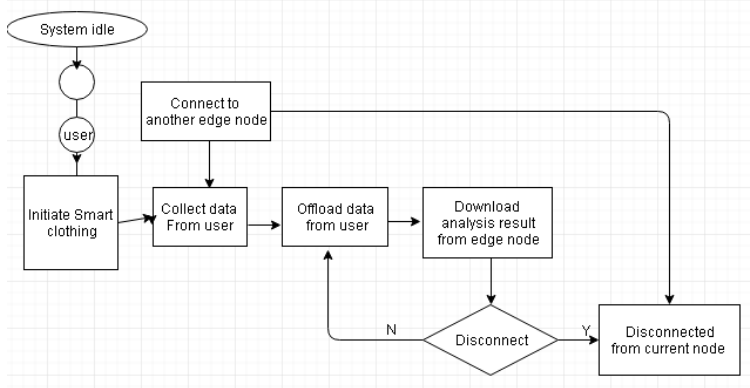

Fig. 8. The flowchart for users to

Offload and download data.

Published By: 
In this ECC based smart healthcare computing system it gives an medical environment to development of application the main goal of this system is to overcome the different resource distribution based on on the current or real time health data collected through the sensors. Next to gives all the medical facility to the patient and analyzed diff rent resource witch need full for the emergency situation of that particular

Patient. In this emergency case where the patient will get immediate treatment and it increases the survival rate of patient with actual diseases.

They having Different scenarios as foll owes Tow patient $\mathrm{X} 1$ and $\mathrm{Y} 1$ both are connected to the edge computing nearest node $\mathrm{A}$ at specific time. the $\mathrm{X} 1$ patient suddenly suffer from emergency disease and immediately he required high amount of medical resources Before coming to the ambulance or any

different resource the Edge A immediately transfer that patient $\mathrm{Y} 1$ to next Edge i.e B and edge A will separately handle $\mathrm{X} 1$ patient. now after that it send the alarm to the hospital and all necessary detail of patient to that hospital or as well as concern doctor after analyzing the problem in that patient doctor will provide medical and proper treatment to the edge

Node and patient will get immediate treatment. Fig. 4 shows a flow diagram for the whole environment.

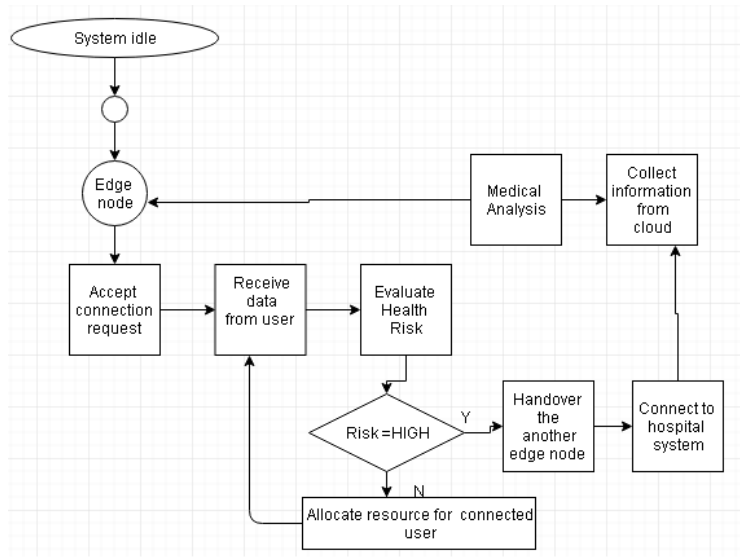

Fig. 9. The flowchart of edge node processing healthcare data..

D. Performance Analysis

To overall the complete performance ECC based healthcare system It mainly focus on the diff rent resource and their application to calculate the complete user performance and Saturation between them are calculated by using some algorithms that is data mining, and deep learning. The performance

has shown as following

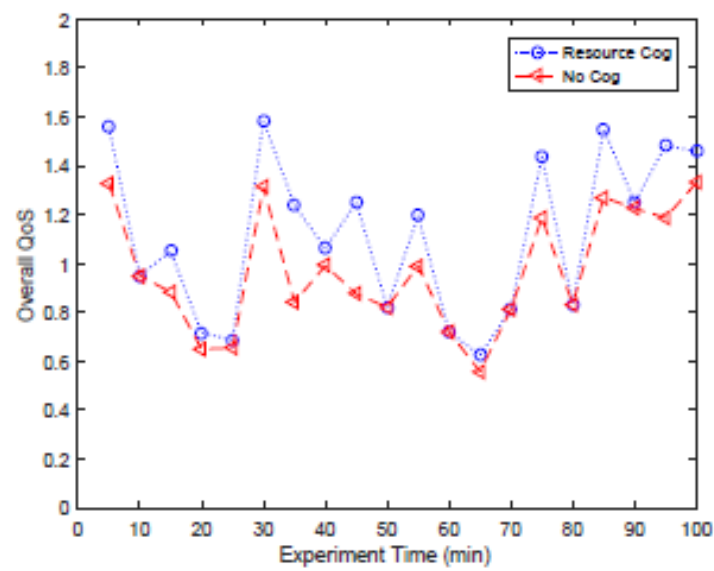

Fig. 10. Overall QoS, with and without the resource cognitive.

Let's consider the E1 number of edge node, and U1 as the number of user after getting the results of that patient it will

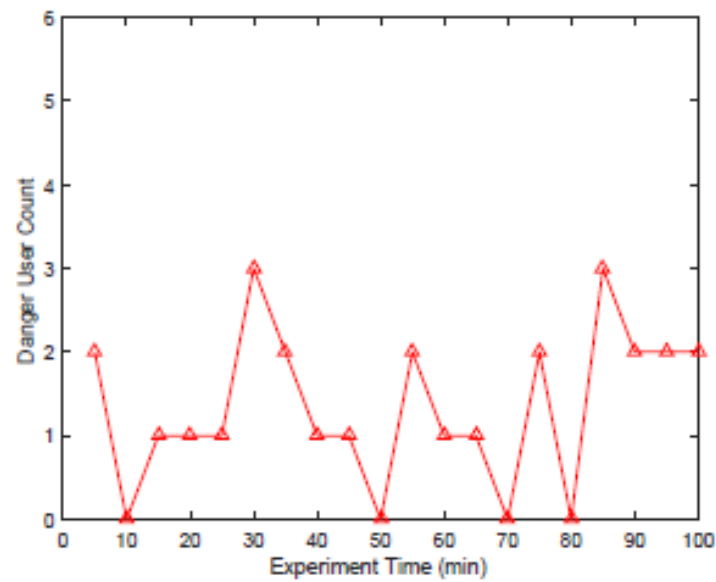

Fig. 11. Danger User Count.

Categorized the risk according health low, medium, high and danger it will distributed them as $0,1,2$, and 3 the health risk level of the particular patient s(i) $20,1,2,3$ and resources are collected the different rates $1 /$ connC((i)), where (i) $2[1, n]$ where (i) $2[1, n]$ i, and $\operatorname{connC}(j), j 2[1, n]$ gives the number of patient recently connected to the edge node $\mathrm{j}$.

$$
\text { Overall QoS }=\sum_{i=1}^{m} \frac{s(i) i}{\operatorname{conn} C(\sigma(i)) i}
$$

The complete explanation has been given to following example lets consider 4 Edge nodes E1, E2, E3, E4 and 5 differ ant users U1, U2, U3, U4, U4, U5. They are all connected to the same resources. All user are in under observation their health status will constantly updated within 2 minutes. all the live updates and real time data which is been miniaturized by doctor and they are all in one network this complete process is QoS. there are two different groups of resource cognition In first group users are connected to the nearest edge node suppose $\mathrm{U} 1, \mathrm{U} 2, \mathrm{U} 3$ are connected to E2 edge all of sudden U2 has some emergency then E2 will separately handle $\mathrm{U} 2$ rest $\mathrm{U} 1$ 
and $\mathrm{U} 3$ will connect to diff rent edges like E3 or E4

like wise then after than second group will be form which will take care of rest users. now the first group is restricted resource of user in emergency state and second group will not allow to enter the first group

As a result of this two diff rent scenario or two diff rent states i.e with cognition result constantly increasing the quality of Qos. as the system distribute the different edge resource once the patient goes into the emergency state it will serve by independently and he will get all facility what they want. After detecting the exact disease of first group of patient appropriate

actions will be taken the complete or overall Qos has been improved. and constantly monitor the patient health statue and it also updates in 2 min so this complete concept will help to become better QoS. The complete health statues has been monitored by using the sensors we are using that temperature heartbeat humidity and pressure this sensor will help out to generate exact situation of patient it will improve the survival rate of the patient.

This Edge cognitive computing real time healthcare system it is need to deploy the explicit cognitive computing in network

edge this communication latency gives large resource for different users.

\section{RESULT GRAPH OF DISEASE}

This Graphical view of Result Evaluation which is showing the graph of increasing Humidity and Temperature instance with the time. If temperature goes on beyond on threshold value the alarm will be generated.

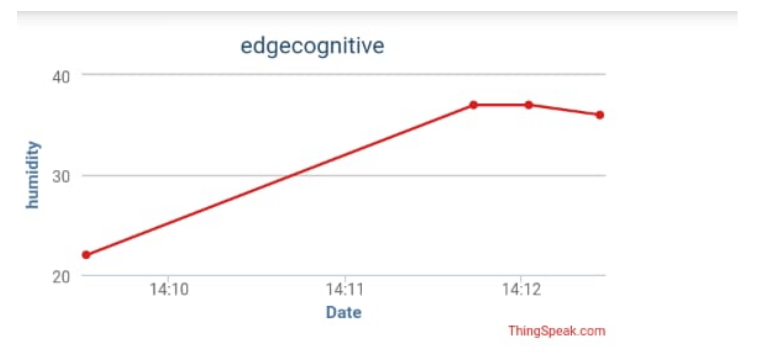

Fig. 12. Evaluation Reference.

\section{CONCLUSION}

All the identical modules like Heartbeat detection module, humidity sensor, temperature sensor etc. and auto sensors are

gives a specified results and different system module also produce optimized result in a circuit most important part here is whatever sensors and circuit we have used in this project are easily available in market as like with the with development in integrated industry Raspberry Pi3 are easily available in market it will help to increasing the processing speed, reduce the power efficiency. it has increased the development healthcare industry. Now this system also comes in smart phone so its big advantage of that. and increasing era of internet in different countries through the mobile phone

with this edge cognitive computing with faster rate it gives the better quality of healthcare industry.

\section{REFERENCES}

1. The Internet of Things in healthcare: an overview https://scholar.google.com/citations?userY4opLB8AAAA

2. Envisioning inclusive futures: technologybased assistive sensory and action substitution https://www.infona.pl/resource/bwmeta1.element.elsevie 3d45bfddfe55- 359f-84e4-674a21 cae024

3. A multiple communication standards compatible IoT system for medical usage http://ieeexplore.ieee.org/document/6577775/?reload=true

4. Ubiquitous data accessing method in IoT-based information system for emergency medical services https://www.deepdyve.com/lp/institute-ofelectrical- and-electronicsengineers/ubiquitous-data-accessing-methodin-iot-based-informationsystem-for- YCZzyY5W9g

5. Implementation of a medical support system considering P2P and IoT technologies

https://www.computer.org/csdl/proceedings/cisis/2014/4325/00/4325a 101- abs.html

6. Acquisition and management of biomedical data using Internet of Things concepts http://ieeexplore.ieee.org/document/7050625/ Real time internet application with distributed ow environment for medical

https://csdl.computer.org/csdl/proceedings/icgciot/2015/7910/00/0738 0578-abs.html

7. Secure end-to-end communication for constrained devices in IoTenabled am- bient assisted living systems https://www.computer.org/csdl/proceedings/wfiot/

2015/0366/00/07389141-abs.html

8. S. Iyengar, F. T. Bonda, R. Gravina, A. Guerrieri, G. Fortino, A. Sangiovanni- Vincen- telli, A framework for creating healthcare monitoring applications using wireless body sensor networks, in Proceedings of the ICST 3rd international conference on Body area networks, ICST (Institute for Computer Sciences, Social-Informatics and Telecommunications Engineering), 2008, p. 8.

9. M. Chen, S. Gonzalez, A. Vasilakos, et al. Body area networks: A survey. Mobile networks and applications, 2011, 16(2): 171-193. 